\title{
Indole-3-acetic acid regulates the central metabolic pathways in Escherichia coli
}

\begin{abstract}
Correspondence
Roberto Defez

defez@igb.cnr.it
\end{abstract}

Received 13 December 2005

Revised 12 April 2006

Accepted 27 April 2006

\author{
C. Bianco, ${ }^{1}$ E. Imperlini, ${ }^{1}$ R. Calogero, ${ }^{2}$ B. Senatore, ${ }^{1}$ P. Pucci $^{3}$ \\ and R. Defez ${ }^{1}$ \\ ${ }^{1}$ Institute of Genetics and Biophysics 'Adriano Buzzati Traverso', via P. Castellino 111, \\ 80131 Naples, Italy \\ 2Dipartimento di Scienze Cliniche e Biologiche, Ospedale S. Luigi, Regione Gonzole 10, \\ Orbassano (TO), Italy \\ ${ }^{3}$ Dipartimento di Chimica Organica e Biochimica, Università Federico II di Napoli, Via Cinthia, \\ 80126 Napoli, Italy
}

The physiological changes induced by indoleacetic acid (IAA) treatment were investigated in the totally sequenced Escherichia coliK-12 MG1655. DNA macroarrays were used to measure the mRNA levels for all the 4290 E. coli protein-coding genes; 50 genes $(1 \cdot 1 \%)$ exhibited significantly different expression profiles. In particular, genes involved in the tricarboxylic acid cycle, the glyoxylate shunt and amino acid biosynthesis (leucine, isoleucine, valine and proline) were up-regulated, whereas the fermentative adhE gene was down-regulated. To confirm the indications obtained from the macroarray analysis the activity of 34 enzymes involved in central metabolism was measured; this showed an activation of the tricarboxylic acid cycle and the glyoxylate shunt. The malic enzyme, involved in the production of pyruvate, and pyruvate dehydrogenase, required for the channelling of pyruvate into acetyl-CoA, were also induced in IAA-treated cells. Moreover, it was shown that the enhanced production of acetyl-CoA and the decrease of NADH/NAD ${ }^{+}$ratio are connected with the molecular process of the IAA response. The results demonstrate that IAA treatment is a stimulus capable of inducing changes in gene expression, enzyme activity and metabolite level involved in central metabolic pathways in E. coli.

\section{INTRODUCTION}

Indoleacetic acid (IAA) is a ubiquitous molecule present in most living organisms and is the main plant growth hormone with auxin activity. IAA plays a key role in the control of many physiological processes in plants, such as cell division, shoot growth, apical dominance, vascular development and tropic response to abiotic stimuli (e.g. light and gravity) (Davies, 1995). It was recently reported that nitric oxide mediates the IAA response through the activation of soluble guanylate cyclase and the following transient increase of cyclic GMP (Pagnussat et al., 2003). IAA also plays a key role in the regulation of 'stringent control', a process mediated by the bacterial alarmone ppGpp, which is produced in the chloroplasts of plant cells in response to stressful conditions (Takahashi et al., 2004). Soil bacteria (such as species of Pseudomonas, Azospirillum, Agrobacterium and Rhizobium) involved in intimate relationship with plants synthesize IAA as a communication

Abbreviations: CRP, cAMP receptor protein; E-D, Entner-Doudoroff; $I A A$, indoleacetic acid; TCA, tricarboxylic acid.

The GEO accession number for the array studies reported in this paper is GSE4941. system with the host plant, and many of them use IAA in pathogenic interactions (tumours, hairy roots, etc.). These bacterial systems mainly synthesize IAA from tryptophan via the indoleacetamide (Lemcke et al., 2000) or indole-3pyruvate (Zimmer et al., 1998) biosynthetic pathways.

IAA is also involved in the morphogenetic development of Saccharomyces cerevisiae. At high concentration, IAA blocks the growth of yeast cells, whereas at lower concentration it induces filamentation and adhesion, factors that are important in plant infection by fungi. These events are mediated by a family of transporters and by the fungalspecific transcription factor Yap1 (Prusty et al., 2004). In mammals IAA is a metabolite of the neurotransmitter serotonin (Artigas et al., 1985). In enteric bacteria IAA is able to compensate for the absence of cAMP, restoring some specific pathways. It is able to replace cAMP in the regulation of the arabinose operon (Ebright \& Beckwith, 1985) and the acetohydroxy acid synthase gene (Williams, 1986) in Escherichia coli. The mechanism of IAA action on the regulation of these genes is unclear. Experiments with two separate classes of small molecules, cAMP and indole derivatives, support the hypothesis of a local denaturation of the DNA helix (Ebright \& Wong, 1981). Thus, IAA appears 
to trigger, in different biological systems, changes in gene regulation leading to different developmental programmes. However, although IAA's role as the main auxin plant hormone has been known for 70 years, its function in eukaryotes and prokaryotes is still largely unexplored. In the present study, we investigated the effect of IAA on the transcript, enzyme and metabolic profile of E. coli, a bacterium not usually exposed to IAA in its natural environment. We demonstrate that IAA activates the tricarboxylic acid (TCA) cycle and the glyoxylate shunt, while it reduces the $\mathrm{NADH} / \mathrm{NAD}^{+}$ratio.

\section{METHODS}

Bacterial growth conditions. Cells were grown aerobically at $37^{\circ} \mathrm{C}$ in $\mathrm{M} 9$ minimal medium containing $20 \mathrm{mg}$ uracil $\mathrm{l}^{-1}, 1 \mathrm{mg}$ thiamine $1^{-1}, 0 \cdot 4 \%$ L-arabinose, glucose, mannitol, lactose or glycerol as carbon source, and supplemented with $40 \mathrm{mg}$ casein acid hydrolysate $1^{-1}$. Solid media contained $15 \mathrm{~g}$ agar (Difco) $1^{-1}$ in TY $(0.5 \%$ yeast extract, $0.8 \% \mathrm{NaCl}$ and $1 \%$ tryptone $)$ or minimal medium. Exponentially growing E. coli K-12 MG1655 cultures $\left(\mathrm{OD}_{600} 0 \cdot 6\right)$ were split into two aliquots; to the first one, an IAA solution was added to a final concentration of $0.5 \mathrm{mM}$ and the other was left untreated (control). After $2 \mathrm{~h}\left(\mathrm{OD}_{600} 1 \cdot 2\right.$ for both cultures) different cell batches, taken from IAA-treated and untreated cells, were aliquoted, frozen in liquid nitrogen for $5 \mathrm{~min}$ and stored at $-80^{\circ} \mathrm{C}$ for use in experiments. Neither the growth rate nor the viability of the $E$. coli cells was affected by $0.5 \mathrm{mM}$ IAA treatment, as already shown by Kline et al. (1980) for another E. coli K-12 strain. At least three independent experiments were performed for all the results presented in this work. When more than three repeated experiments were done, the number of repetitions is indicated in the table footnote or figure legend.

RNA isolation. Total RNA was purified from cells grown in M9 arabinose medium using the RNeasy Mini-Kit from Qiagen and following the manufacturer's instructions. The isolated RNA was incubated at $37^{\circ} \mathrm{C}$ with $5 \mathrm{U}$ DNase I (Promega) and $40 \mathrm{U}$ RNasin ribonuclease inhibitor (Promega) for $30 \mathrm{~min}$, extracted with phenol/ chloroform, precipitated with 2.5 vols ethanol and then dissolved in diethylpyrocarbonate-treated water. After purification, the RNA concentration was determined by absorbance at $260 \mathrm{~nm}$ and the RNA was stored at $-20^{\circ} \mathrm{C}$ until further use.

Probe synthesis. ${ }^{33} \mathrm{P}$-labelled probes were prepared using E. coli gene-specific primers (Sigma-Genosys) following the protocol provided by the manufacturer, except that the reaction mixture contained $40 \mu \mathrm{Ci}(1.48 \mathrm{MBq})\left[\alpha_{-}{ }^{33} \mathrm{P}\right] \mathrm{dCTP}\left(1000-3000 \mathrm{Ci} \mathrm{mmol}{ }^{-1}\right.$; Amersham Pharmacia), and $200 \mathrm{U}$ Superscript II (Promega) in a $30 \mu \mathrm{l}$ volume. Unincorporated radiolabelled nucleotides were removed by applying the reaction mixture to a Sephadex G-25 gel filtration spin column.

Array hybridization. The DNA arrays (Panorama E. coli gene arrays) used in the hybridization experiments were produced by Sigma-Genosys Biotechnologies. Each DNA array consists of a $12 \times 24 \mathrm{~cm}$ positively charged nylon membrane on which $10 \mathrm{ng}$ each of all 4290 PCR-amplified ORF-specific DNA fragments are robotically printed in duplicate. The hybridization and washing steps were carried out as described by the manufacturer. Briefly, the nylon membranes were pre-hybridized in $5 \mathrm{ml}$ hybridization buffer [ $5 \times$ SSPE $\left(1 \times\right.$ SSPE is $0.18 \mathrm{M} \mathrm{NaCl}, 10 \mathrm{mM} \mathrm{NaH}{ }_{2} \mathrm{PO}_{4}$ and $1 \mathrm{mM}$ EDTA, pH 7·7), $2 \%$ SDS, $1 \times$ Denhardt's reagent and $100 \mu \mathrm{g}$ denatured, sonicated salmon sperm DNA per ml]. After incubation for $2 \mathrm{~h}$ at $65^{\circ} \mathrm{C}$, the pre-hybridization solution was replaced with $3 \mathrm{ml}$ hybridization buffer containing the denatured radioactively labelled cDNA probes. Filter hybridizations were carried out for $16 \mathrm{~h}$ in a hybridization oven. The filters were then washed three times, for $5 \mathrm{~min}$ each, at room temperature with $50 \mathrm{ml}$ wash solution $(0.5 \times$ SSPE, $0.2 \%$ SDS) and then three times at $65^{\circ} \mathrm{C}$ with $100 \mathrm{ml}$ warmed wash solution. The washed filters were blotted between two pieces of Whatman $3 \mathrm{MM}$ paper for $5 \mathrm{~min}$ and then wrapped in Saran Wrap. For quantification, filters were exposed to a phosphorimager screen. The blots were stripped at $100{ }^{\circ} \mathrm{C}$ with $1 \%$ SDS in Tris/EDTA buffer as specified by the manufacturer.

Data analysis. The exposed phosphorimager screens were scanned at $100 \mu \mathrm{m}$ resolution using a STORM 445 PhosphorImager (Molecular Dynamics). The resulting files were analysed to determine the signal intensities for each spot by summing the value of each pixel within the boundaries of the spot using ImageQuant (version 3.0) software (Molecular Dynamics). Background values were measured in the surrounding region of the four corner grids containing E. coli genomic DNA, as a positive control, and empty spots. The intensity values for each spot were exported from ImageQuant to Microsoft Excel. Each ORF-specific spot was present in duplicate, and the mean of the normalized intensity values of the duplicate spots of each gene was used for further analysis. To avoid extreme intensity ratio for genes close to or below the detection limit, signal intensity values corresponding to a signal-to-background (S/B) ratio $<2 \cdot 0$ were scaled up to a value corresponding to the normalized background means. To identify ORFs whose expression was altered by addition of IAA, the two growth conditions (with and without IAA addition) were compared by determining the ratio of the corresponding intensities of each pair of ORF-specific spots on two blots. These ratios represent the relative transcript levels of each E. coli ORF under the same growth condition. Ratios were calculated such that the $\log _{2}$ of the absolute value of the expression ratio was positive for intensities that were higher in IAA-treated cells and negative for intensities that were higher in control cells. A gene was considered significantly regulated when the relative intensity was least two times greater than the background expression threshold and the $\log _{2}$ expression ratio was $\geqslant 0 \cdot 8$ (up-regulated) or $\leqslant-0 \cdot 8$ (down-regulated) and more than 4 times standard deviation from the mean.

Real-time PCR studies. Total RNA was isolated from cells grown in M9 arabinose medium using a RNeasy Mini Kit (Qiagen) and following the manufacturer's protocol. Residual DNA present in the RNA preparations was removed by RNase-free DNase I treatment (Epicentre Technologies). cDNAs were synthesized with the StrataScript reverse transcription reagents (Stratagene) and random hexamers as primers. One 'no RT' control (without reverse transcriptase) for each RNA sample and one 'no RNA' control (replacing RNA with distilled $\mathrm{H}_{2} \mathrm{O}$ ) for each primer and probe set were also performed. Specific primer pairs were designed using the Primer3 software. Primers for rrsA of the $16 \mathrm{~S}$ rRNA gene were also designed, and this gene was included in all the Q-RT-PCR analyses for data normalization. Real-time PCR was performed with each specific primer pair by using the DyNamo HS SYBR Green qPCR kit (Finnzymes). The reactions were performed with the DNA Engine OPTICON 2 system (MJ Research). RT-PCR amplification for each cDNA sample was performed in triplicate wells. During the reactions the fluorescence signal due to SYBR Green intercalation was monitored to quantify the double-stranded DNA product formed in each PCR cycle. Results were recorded as relative gene expression changes after normalizing for $r r s A$ gene expression and computed using the comparative $C_{\mathrm{T}}$ method $\left(2^{-\Delta \Delta \mathrm{CT}}\right)$ described in detail by Livak \& Schmittgen (2001).

Metabolite determination. For this analysis the cells were grown in $\mathrm{M} 9$ arabinose medium. The level of $\mathrm{NADH}$ and $\mathrm{NAD}^{+}$was measured by first extracting the nucleotides from cells harvested at 
different growth stages and then assaying for the two metabolites as described by Zhang et al. (2000) by using alcohol dehydrogenase to convert $\mathrm{NAD}^{+}$to $\mathrm{NADH}$ and lactate dehydrogenase to convert $\mathrm{NADH}$ to $\mathrm{NAD}^{+}$. For intracellular acetyl-CoA determination the extraction and assay was based on the method described by Pruss \& Wolfe (1994) with some modifications. E. coli cells $(40 \mathrm{ml})$ were centrifuged, resuspended in $4 \mathrm{ml}$ washing buffer $[10 \mathrm{mM}$ sodium phosphate (pH 7.5), $10 \mathrm{mM} \mathrm{MgCl}_{2}$ and $1 \mathrm{mM}$ EDTA] and treated with $0.8 \mathrm{ml} 3 \mathrm{M}$ ice-cold $\mathrm{HClO}_{4}$. After rapid vortexing, the mixture was kept in ice for $30 \mathrm{~min}$. Cell debris was removed from the mixture by centrifugation at $2800 \mathrm{~g}$ for $5 \mathrm{~min}$. The $\mathrm{HClO}_{4}$ in the supernatant was neutralized immediately with $1.3 \mathrm{ml}$ of $3 \mathrm{M} \mathrm{KHCO}_{3}$. The resulting $\mathrm{HClO}_{4}$ precipitate was removed by further centrifugation at $8000 \mathrm{~g}$ for $10 \mathrm{~min}$. A $0.5 \mathrm{ml}$ sample of this neutralized extract was assayed for acetyl-CoA by using a coupled enzyme assay with malate dehydrogenase and citrate synthase (Pruss \& Wolfe, 1994), and acetyl-CoA was measured indirectly by monitoring the reduction of $\mathrm{NAD}^{+}$to NADH. The intracellular acetyl-CoA concentration was expressed in $\mathrm{mM}$ using a measured cell density of $5 \times 10^{11}$ cells per 1 per $\mathrm{OD}_{600}$ unit and an approximated cell volume of $3 \times 10^{-15} 1$ per cell (Chohnan et al., 1998). The extracellular levels of acetate and pyruvate were measured by a coupled reaction with acetate kinase and phosphotransacetylase (Pruss \& Wolfe, 1994) and by a colorimetric assay (Patnaik et al., 1992), respectively. The concentrations were expressed in $\mathrm{mM}$.

Preparation of cell extracts. Harvested cells were dissolved in extraction buffer $(20 \mathrm{mM}$ Tris/ $\mathrm{HCl}, \mathrm{pH} 8 \cdot 0$, containing $1 \mathrm{mM}$ EDTA, $20 \mathrm{mM} \mathrm{KCl}, 1 \mathrm{mM}$ DTT, $10 \%$, v/v, glycerol and $1 \mathrm{mM}$ PMSF) and then disrupted by sonication (MSE $150 \mathrm{~W}$ ) for $2 \mathrm{~min}$ (in $30 \mathrm{~s}$ periods). Cell debris was removed by centrifugation at $16000 \mathrm{~g}$ (Eppendorf $5415 \mathrm{D}$ ) for $10 \mathrm{~min}$; the resulting crude cellfree extracts were immediately used for determination of specific enzyme activities or stored at $-20^{\circ} \mathrm{C}$. Protein concentrations were determined by the Bradford assay, using BSA as a standard.

Enzyme activity measurement. The activity of 34 enzymes was monitored in M9 arabinose medium for control and IAA-treated cells cultured at the same growth stage as for mRNA analysis. Three of these enzymes, involved in the TCA cycle and the glyoxylate shunt (citrate synthase, isocitrate dehydrogenase and malate synthase), were selected to assay their activity in cells grown with five different carbon sources (arabinose, glucose, mannitol, lactose and glycerol).

Previously published methods were used to assay the activity of the overall Entner-Doudoroff (E-D) pathway enzymes [including 6-phosphogluconate dehydratase (Edd) and 2-keto-3-deoxy-6phosphogluconate aldolase (Eda)] (Alleanza \& Lessie, 1982), ribose phosphate isomerase (RpiA) (Hove-Jensen \& Maigaard, 1993), transketolase (TktA) (Josephson \& Fraenkel, 1969), transaldolase (TalA) (Tchola \& Horecker, 1966), phosphotransacetylase (Pta) (Brown et al., 1977), acetyl-CoA synthetase (Acs) (Brown et al., 1977), acetate kinase (Ack) (Skarstedt \& Silverstein, 1976), alcohol dehydrogenase (AdhE) (Clark \& Cronan, 1980), lactate dehydrogenase (LdhA) (Kochhar et al., 1992), phosphoglucose isomerase (Pgi) (Reithel, 1966), phosphofructokinase (Pfk) (Ling et al., 1969), fructose bisphosphate aldolase (Fba) (Rutter et al., 1969), triosephosphate isomerase (TpiA) (Eikmanns, 1992), glyceraldehyde-3-phosphate dehydrogenase (GAPDH) (Eikmanns, 1992), phosphoglycerate kinase (Pgk) (D’alessio \& Josse, 1966), phosphoglyceromutase (PgmA) (D'alessio \& Josse, 1966), enolase (Eno) (Westhead, 1966), pyruvate kinase (Pyk) (Valentine \& Tanaka, 1966), fructose-1,6bisphosphatase (Fbp) (Donahue et al., 2000), phosphoenolpyruvate carboxylase (Ppc) (Wohl \& Markus, 1972), pyruvate dehydrogenase (AceE) (Schartz et al., 1968), phosphoenolpyruvate synthetase (PpsA) (Cooper \& Kornberg, 1966), malic enzyme (SfcA) (Stols \& Donnelly, 1997), citrate synthase (GltA) (Weitzman, 1969), aconitase (AcnA)
(Fansler \& Lowenstein, 1969), isocitrate dehydrogenase (IcdA) (MuroPastor \& Florencio, 1992), 2-oxoglutarate dehydrogenase (SucAB) (Reed \& Mukherjee, 1969), succinyl-CoA synthetase (SucCD) (Bridger et al., 1969), succinate dehydrogenase (SdhAB) (King, 1969), fumarase (FumA) (Hill \& Bradshaw, 1969), malate dehydrogenase (Mdh) (Kitto, 1969), isocitrate lyase (AceA) (McFadden, 1969), malate synthase (AceB) (Dixon \& Kornberg, 1969), and acetohydroxy acid synthase (IlvB) (De Felice et al., 1988).

Each reaction was started by the addition of enzyme extract and the activity was measured spectrophotometrically in a Beckman Coulter recording spectrophotometer. The wavelength and the millimolar absorption coefficients for $\mathrm{NAD}^{+}, \mathrm{NADH}, \mathrm{NADP}^{+}$and $\mathrm{NADPH}$ were $340 \mathrm{~nm}$ and $6 \cdot 22 \mathrm{~cm}^{-1} \mathrm{mM}^{-1}$, respectively. One unit of specific enzyme activity is defined as the amount of enzyme required to convert $1 \mu \mathrm{mol}$ substrate per min per mg protein under the published reaction conditions. The control cultures were used as a reference for comparison.

\section{RESULTS}

\section{Gene expression profiling of control and IAA- treated $E$. coli cells}

Gene expression levels for control and IAA-treated cells were monitored by using DNA macroarray membranes containing the whole E. coli K-12 MG1655 genome. Membranes had duplicated spots for each gene and each experiment was repeated three times, yielding six datasets. We found 50 genes with expression profiles significantly altered (Table 1). The mRNA levels of 41 genes increased and that of 9 genes decreased in IAA-treated cells as compared to controls.

When the differentially expressed genes were further classified into groups by their function (Table 1) we found that $50 \%$ of the up-regulated genes were associated with energy metabolism and amino acid biosynthesis.

\section{Carbon and energy metabolism}

Several genes involved in the TCA cycle (gltA, sucA, sucD, $s d h B$ and $s d h C$ ) were up-regulated in IAA-treated cells. In addition, the expression of the aceA gene, encoding isocitrate lyase, involved in the isocitrate catabolic pathway at the branch point between the glyoxylate shunt and the TCA cycle, and of the cyoD gene, encoding a subunit of the aerobic respiratory system, cytochrome bo terminal oxidase complex, was higher in treated cells.

The expression of several carbon-transport genes, such as the arginine $\mathrm{ABC}$ transport gene art $\mathrm{Q}$ and the ribose-uptake gene $r b s C$, which are regulated by catabolite repression, was affected by IAA treatment. In addition, the mlc gene, which encodes the global regulator (repressor) Mlc, involved in glucose uptake (Shin et al., 2001), and glpK, encoding a glycerol kinase involved in the utilization of glycerol as carbon source (Weissenborn et al., 1992), were also induced in IAA-treated cells. In contrast, the expression level of the uhpA gene, which regulates the induction of the sugar-phosphate transport system (Insland et al., 1992), and of the adhE gene, encoding the fermentative enzyme 
Table 1. E. coli MG1655 genes whose relative expression level increased or decreased after treatment with $0.5 \mathrm{mM} I A A$

\begin{tabular}{|c|c|c|c|}
\hline Gene & Known or predicted function & Functional classification ${ }^{\star}$ & Fold change $\dagger$ \\
\hline leuD & 3-Isopropylmalate isomerase & Metabolism & $2 \cdot 0 \pm 0 \cdot 2$ \\
\hline$b 0725$ & Unknown CDS & Unclassified & $2 \cdot 0 \pm 0 \cdot 2$ \\
\hline sucA & 2-Oxoglutarate dehydrogenase & Metabolism & $1 \cdot 6 \pm 0 \cdot 3$ \\
\hline gltA & Citrate synthase & Metabolism & $1 \cdot 5 \pm 0 \cdot 2$ \\
\hline sucD & Succinyl-CoA synthetase & Metabolism & $1 \cdot 5 \pm 0 \cdot 3$ \\
\hline$s d h B$ & Succinate dehydrogenase & Metabolism & $1 \cdot 4 \pm 0 \cdot 2$ \\
\hline pspA & Negative regulatory gene for the $p s p$ operon & Cell processes & $1 \cdot 4 \pm 0 \cdot 3$ \\
\hline$y c h N$ & Putative phosphatase & Unclassified & $1 \cdot 3 \pm 0 \cdot 2$ \\
\hline proA & $\gamma$-Glutamylphosphate reductase & Metabolism & $1 \cdot 2 \pm 0 \cdot 2$ \\
\hline$b 4341$ & Unknown CDS & Unclassified & $1 \cdot 2 \pm 0 \cdot 2$ \\
\hline aceA & Isocitrate lyase & Metabolism & $1 \cdot 2 \pm 0 \cdot 2$ \\
\hline$y z g L$ & Conserved protein & Unclassified & $1 \cdot 2 \pm 0 \cdot 2$ \\
\hline$c y o D$ & Cytochrome $o$ ubiquinol oxidase subunit IV & Metabolism & $1 \cdot 1 \pm 0 \cdot 1$ \\
\hline$m l c$ & Transcriptional repressor for glucose uptake and glycolysis & Metabolism & $1 \cdot 1 \pm 0 \cdot 2$ \\
\hline b1321 & Conserved hypothetical protein & Unclassified & $1 \cdot 1 \pm 0 \cdot 2$ \\
\hline fliY & Cysteine-binding periplasmic transport protein & Cell processes & $1 \cdot 1 \pm 0 \cdot 2$ \\
\hline artQ & Arginine 3 rd transport system & Cell processes & $1 \cdot 1 \pm 0 \cdot 2$ \\
\hline$i l v D$ & Dihydroxyacid dehydratase & Metabolism & $1 \cdot 1 \pm 0 \cdot 2$ \\
\hline caiE & Putative acyl transferase & Metabolism & $1 \cdot 1 \pm 0 \cdot 2$ \\
\hline $\operatorname{exbB}$ & Uptake of enterochelin & Cell processes & $1 \cdot 1 \pm 0 \cdot 2$ \\
\hline$c y c A$ & D-Alanine/D-serine/glycine transport protein & Metabolism & $1 \cdot 1 \pm 0 \cdot 2$ \\
\hline $\operatorname{san} A$ & Vancomycin sensitivity & Cell processes & $1 \cdot 0 \pm 0 \cdot 1$ \\
\hline$b 2915$ & Conserved hypothetical protein & Unclassified & $1 \cdot 0 \pm 0 \cdot 1$ \\
\hline fdoG & Formate dehydrogenase $\mathrm{O}$, major subunit & Metabolism & $1 \cdot 0 \pm 0 \cdot 1$ \\
\hline$d n a K$ & Chaperone Hsp70 & Cell processes & $1 \cdot 0 \pm 0 \cdot 2$ \\
\hline $\operatorname{rhlB}$ & Putative ATP-dependent helicase & Unclassified & $1 \cdot 0 \pm 0 \cdot 2$ \\
\hline$y d e W$ & Putative transcriptional regulator (repressor) & Transcription & $1 \cdot 0 \pm 0 \cdot 2$ \\
\hline $\operatorname{leu} B$ & 3-Isopropylmalate dehydrogenase & Metabolism & $1 \cdot 0 \pm 0 \cdot 2$ \\
\hline$h t p G$ & Chaperone Hsp90 & Cell processes & $1 \cdot 0 \pm 0 \cdot 2$ \\
\hline$m c r C$ & Component of 5-methylcytosine-specific restriction enzyme McrBC & Metabolism & $1 \cdot 0 \pm 0 \cdot 2$ \\
\hline pstB & High-affinity phosphate transporter & Metabolism & $1 \cdot 0 \pm 0 \cdot 2$ \\
\hline relB & Transcriptional repressor of relBE operon & Cell processes & $1 \cdot 0 \pm 0 \cdot 2$ \\
\hline$s d h C$ & Succinate dehydrogenase & Metabolism & $1 \cdot 0 \pm 0 \cdot 2$ \\
\hline$g l p K$ & Glycerol kinase & Metabolism & $1 \cdot 0 \pm 0 \cdot 2$ \\
\hline $\mathrm{rbs} C$ & D-Ribose high-affinity transport protein & Metabolism & $0 \cdot 9 \pm 0 \cdot 2$ \\
\hline gals & Transcriptional repressor for galactose utilization & Metabolism & $0 \cdot 9 \pm 0 \cdot 2$ \\
\hline$i l v E$ & Branched-chain amino-acid aminotransferase & Metabolism & $0 \cdot 9 \pm 0 \cdot 1$ \\
\hline$y f h E$ & Co-chaperone protein $\mathrm{Hsc} 20$ & Cell processes & $0 \cdot 9 \pm 0 \cdot 1$ \\
\hline yrfI & Heat-shock protein 33 & Cell processes & $0 \cdot 8 \pm 0 \cdot 1$ \\
\hline$f a b D$ & Malonyl-CoA-[acyl-carrier-protein] transacylase & Metabolism & $0 \cdot 8 \pm 0 \cdot 1$ \\
\hline$b 1279$ & Conserved hypothetical protein & Unclassified & $0 \cdot 8 \pm 0 \cdot 2$ \\
\hline galR & Transcriptional repressor for galactose utilization & Metabolism & $-0 \cdot 9 \pm 0 \cdot 1$ \\
\hline b1362 & Putative prophage lambda endopeptidase & Phage related & $-0 \cdot 9 \pm 0 \cdot 2$ \\
\hline$y a g W$ & Conserved protein & Unclassified & $-1 \cdot 0 \pm 0 \cdot 1$ \\
\hline $\operatorname{nanR}$ & Trancriptional repressor of the nan operon & Transcription & $-1 \cdot 0 \pm 0 \cdot 1$ \\
\hline$y j i Y$ & Carbon starvation protein & Cell processes & $-1 \cdot 1 \pm 0 \cdot 2$ \\
\hline $\operatorname{arcD}$ & Arginine/ornithine antiporter & Cell processes & $-1 \cdot 1 \pm 0 \cdot 2$ \\
\hline$y h j X$ & Putative oxalate/formate antiporter & Cell processes & $-1 \cdot 1 \pm 0 \cdot 2$ \\
\hline$a d h E$ & Alcohol dehydrogenase & Metabolism & $-1 \cdot 1 \pm 0 \cdot 2$ \\
\hline$u h p A$ & Response regulator (activator) & Metabolism & $-1 \cdot 1 \pm 0 \cdot 2$ \\
\hline
\end{tabular}

${ }^{\star}$ Based on the GenProtEC database (http//genprotec.mbl.edu/).

$\dagger \log _{2}$ (expression ratio) of measured transcript levels determined for IAA-treated and untreated cells; the value is positive for genes that are more expressed in IAA-treated cells and negative for genes more highly expressed in control cells. 
alcohol dehydrogenase, induced under anaerobic conditions, was severely down-regulated in IAA-treated cells. Gene members of several operons (cyoABCDE, ilvGMEDA, sucABCD, sdhCBAD, proBA, dnaKJ and leuABCD) were regulated in a coordinate way; however, not all the genes of these operons were included in Table 1 either because of a high standard deviation or because of a $\log _{2}$ expression ratio below $\pm 0 \cdot 8$. For the above reasons we did not find the $\operatorname{araBAD}$ operon and the $i l v B$ gene among the genes significantly regulated, although they were both slightly induced. The ara operon and the $i l v B$ gene were previously described as induced by IAA treatment (Ebright \& Beckwith, 1985; Williams, 1986).

\section{Biosynthetic pathways}

Genes involved in amino acid biosynthesis, in particular those involved in the branched-chain amino acid biosynthetic pathways, were significantly induced in IAA-treated cells. Among them we found two genes of the ilvGMEDA operon, which encodes the enzymes for isoleucine and valine synthesis, and two genes of the leuABCD operon, the products of which are required for leucine biosynthesis. In addition, expression of the pro $A$ gene, which encodes $\gamma$ glutamyl phosphate reductase, involved in the biosynthesis of proline, was up-regulated in IAA-treated cells.

\section{Confirmation of the macroarray results by real-time} PCR. A mixture of six upregulated and downregulated metabolic genes (sucA, sucD, gltA, aceA, adhE and galR), was selected for real-time PCR studies. The results of this analysis confirmed those obtained with macroarray hybridizations, although the absolute values of fold changes were different (Table 2), probably due to the different sensitivity of these two techniques.

\section{Enzyme assays}

In order to test whether the transcriptome analysis correlated with the corresponding enzyme activity, we assayed 34 enzymes involved in the main metabolic pathways (Table 3, Table 4 and Fig. 1). We found that most of the TCA cycle activities were significantly induced following IAA treatment. At the important control point of the junction between the TCA cycle and the glyoxylate shunt, isocitrate lyase (AceA) and malate synthase (AceB) were also induced in IAA-treated cells. The activities of several glycolytic enzymes (Pgi, GapA, Pgk, Eno, Pyk and the anaplerotic Ppc) were only slightly increased or unregulated following IAA treatment. Simultaneously, the activities of the gluconeogenic enzymes PpsA and Fbp were reduced and unaffected, respectively. In addition, the pyruvate dehydrogenase (aceEF operon product) and malic enzyme ( $s f A$ gene product) activities were both induced in IAA-treated cells. We could not find a significant alteration of the enzyme activities involved in the oxidative pentose phosphate pathway, including ribose-5-phosphate isomerase (RpiA), transketolase (TktA) and transaldolase (TalA), and the E-D pathway, including 6-phosphogluconate dehydratase (Edd) and 2-keto-3-deoxy-6-phosphogluconate aldolase (Eda). Among the fermentative enzymes, the activity of lactate dehydrogenase (LdhA) was not influenced by IAA treatment, while the activity of alcohol dehydrogenase (AdhE) was reduced. The activity of the enzymes Pta and AckA, involved in acetate synthesis, was unregulated and induced, respectively, whereas that of Acs, involved in acetate consumption, was reduced. Finally, the activity of acetohydroxy acid synthase (IlvB), catalysing the common first step of isoleucine and valine biosynthesis, increased up to $40 \%$ after IAA treatment (Table 3).

To compare the effects of IAA treatment on enzyme activity in cells grown with different carbon sources we selected the enzymes citrate synthase, isocitrate dehydrogenase and malate synthase. The activity of these enzymes was unchanged or slightly induced after IAA treatment when using glucose, mannitol, lactose and glycerol as a carbon source (Table 4). When the cells were grown in arabinose minimal medium the ratio of enzyme activities in IAA-treated versus control cells was more consistently increased. This increased ratio might be explained by the high specificity of IAA for the $\operatorname{araBAD}$ operon as previously reported (Ebright \& Beckwith, 1985).

We found an excellent correlation between gene induction (gltA, aceA, sdhC, sdhB, sucA) and the activation of the corresponding enzymes. Similarly adhE was repressed along with the corresponding enzyme AdhE. However, there

Table 2. Real-time PCR anaylsis

\begin{tabular}{|llc|}
\hline Gene & \multicolumn{1}{c|}{ Known function } & Relative level $^{*}$ \\
\hline sucA & 2-Oxoglutarate dehydrogenase & $2 \cdot 2 \pm 0 \cdot 1$ \\
gltA & Citrate synthase & $1 \cdot 9 \pm 0 \cdot 1$ \\
ace A & Isocitrate lyase & $1 \cdot 8 \pm 0 \cdot 1$ \\
sucD & Succinyl-CoA synthetase & $1 \cdot 4 \pm 0 \cdot 1$ \\
adhE & Alcohol dehydrogenase & $0 \cdot 6 \pm 0 \cdot 1$ \\
galR & Transcriptional repressor for galactose utilization & $0 \cdot 6 \pm 0 \cdot 1$ \\
\hline
\end{tabular}

${ }^{\star}$ Relative gene expression levels from comparative $C_{\mathrm{T}}$ method; $2^{-\Delta \Delta \mathrm{CT}}>1$, gene more highly expressed in IAA-treated cells; $2^{-\Delta \Delta C T}<1$, gene more highly expressed in control cells. 
Table 3. Activity of the main metabolic pathway enzymes

\begin{tabular}{|c|c|c|c|}
\hline \multirow[t]{2}{*}{ Enzyme } & \multicolumn{3}{|c|}{ Specific activity ${ }^{\star}$} \\
\hline & Control & IAA-treated & Ratio $\dagger$ \\
\hline Phosphoglucose isomerase (Pgi) & $2 \cdot 5 \pm 0 \cdot 4$ & $3 \cdot 2 \pm 0 \cdot 5$ & $1 \cdot 28$ \\
\hline Phosphofructokinase (Pfk) & $4 \cdot 9 \pm 0 \cdot 3$ & $4 \cdot 0 \pm 0 \cdot 4$ & $0 \cdot 82$ \\
\hline Fructose bisphosphate aldolase (Fba) & $3 \cdot 0 \pm 0 \cdot 3$ & $2 \cdot 5 \pm 0 \cdot 1$ & $0 \cdot 83$ \\
\hline Triosephosphate isomerase (TpiA) & $132 \pm 7$ & $114 \pm 9$ & $0 \cdot 86$ \\
\hline Glyceraldehyde-3-P dehydrogenase (GapA) & $140 \pm 25$ & $230 \pm 60$ & $1 \cdot 64$ \\
\hline Phosphoglycerate kinase (Pgk) & $5 \cdot 6 \pm 1 \cdot 0$ & $5 \cdot 3 \pm 0 \cdot 6$ & $0 \cdot 95$ \\
\hline Phosphoglyceromutase (PgmA) & $15 \cdot 1 \pm 2 \cdot 8$ & $10 \cdot 2 \pm 2 \cdot 5$ & $0 \cdot 62$ \\
\hline Enolase (Eno) & $0 \cdot 88 \pm 0 \cdot 11$ & $0 \cdot 99 \pm 0 \cdot 18$ & $1 \cdot 11$ \\
\hline Pyruvate kinase (Pyk) & $8 \cdot 8 \pm 0 \cdot 7$ & $10 \cdot 5 \pm 0 \cdot 5$ & $1 \cdot 19$ \\
\hline Fructose-1,6-bisphosphatase (Fbp) & $0 \cdot 29 \pm 0 \cdot 01$ & $0 \cdot 29 \pm 0$ & $1 \cdot 0$ \\
\hline Phosphoenolpyruvate carboxylase (Ppc) & $2 \cdot 9 \pm 0 \cdot 3$ & $3 \cdot 1 \pm 0 \cdot 5$ & $1 \cdot 1$ \\
\hline Pyruvate dehydrogenase (AceE) & $0 \cdot 024 \pm 0 \cdot 001$ & $0 \cdot 036 \pm 0 \cdot 002$ & $1 \cdot 5$ \\
\hline Phosphoenolpyruvate synthetase (PpsA) & $0 \cdot 0030 \pm 0 \cdot 0008$ & $0 \cdot 0019 \pm 0 \cdot 0001$ & $0 \cdot 63$ \\
\hline Malic enzyme (SfcA) & $3 \cdot 8 \pm 0 \cdot 4$ & $5 \cdot 3 \pm 0 \cdot 6$ & $1 \cdot 4$ \\
\hline E-D pathway (Edd-Eda) & $26 \pm 3$ & $28 \pm 4$ & $1 \cdot 1$ \\
\hline Ribose-5-phosphate isomerase (RpiA) & $55 \pm 3$ & $54 \pm 5$ & $0 \cdot 98$ \\
\hline Transketolase (TktA) & $3 \cdot 4 \pm 0 \cdot 6$ & $3 \cdot 4 \pm 0 \cdot 5$ & $1 \cdot 0$ \\
\hline Transaldolase (TalA) & $29 \pm 2$ & $29 \pm 1$ & $1 \cdot 0$ \\
\hline Phosphotransacetylase (Pta) & $277 \pm 43$ & $275 \pm 10$ & $0 \cdot 99$ \\
\hline Acetyl-CoA synthetase (Acs) & $0 \cdot 91 \pm 0 \cdot 1$ & $0 \cdot 65 \pm 0 \cdot 15$ & $0 \cdot 71$ \\
\hline Acetate kinase (Ack) & $0 \cdot 47 \pm 0 \cdot 1$ & $0 \cdot 66 \pm 0 \cdot 2$ & $1 \cdot 4$ \\
\hline Alcohol dehydrogenase (AdhE) & $0 \cdot 48 \pm 0 \cdot 08$ & $0 \cdot 31 \pm 0 \cdot 01$ & $0 \cdot 65$ \\
\hline Lactate dehydrogenase (LdhA) & $43 \pm 3$ & $45 \pm 5$ & $1 \cdot 1$ \\
\hline Aconitase (AcnA) & $0 \cdot 012 \pm 0 \cdot 001$ & $0 \cdot 023 \pm 0 \cdot 003$ & $1 \cdot 9$ \\
\hline 2-Oxoglutarate dehydrogenase (SucAB) & $0 \cdot 046 \pm 0 \cdot 008$ & $0 \cdot 063 \pm 0 \cdot 009$ & $1 \cdot 4$ \\
\hline Succinyl-CoA synthetase (SucCD) & $0 \cdot 12 \pm 0 \cdot 02$ & $0 \cdot 078 \pm 0 \cdot 008$ & $0 \cdot 65$ \\
\hline Succinate dehydrogenase (SdhAB) & $0 \cdot 025 \pm 0 \cdot 006$ & $0 \cdot 030 \pm 0 \cdot 008$ & $1 \cdot 2$ \\
\hline Fumarase (FumA) & $0 \cdot 18 \pm 0 \cdot 01$ & $0 \cdot 25 \pm 0 \cdot 04$ & $1 \cdot 4$ \\
\hline Malate dehydrogenase (Mdh) & $0 \cdot 059 \pm 0 \cdot 004$ & $0 \cdot 057 \pm 0 \cdot 003$ & $0 \cdot 97$ \\
\hline Isocitrate lyase (AceA) & $2 \cdot 1 \pm 0 \cdot 2$ & $3 \cdot 2 \pm 0 \cdot 4$ & $1 \cdot 5$ \\
\hline Acetohydroxy acid synthase (IlvB) & $3 \cdot 0 \pm 0 \cdot 3$ & $4 \cdot 2 \pm 0 \cdot 5$ & $1 \cdot 4$ \\
\hline
\end{tabular}

${ }^{*}$ The unit of enzyme activity is $\mu \mathrm{mol} \mathrm{min}^{-1}$ (mg protein $)^{-1}$.

$\dagger$ Ratio calculation was based on the control condition. The values reported in the table are the means $\pm \mathrm{SD}$ of at least five measurements.

was a major exception in the consistency among gene and enzyme regulation concerning the $s u c D$ gene (up-regulated) and the relative enzyme activity of succinyl-CoA synthetase (down-regulated). The sucD gene is the last member of the $P_{s d h} s d h C D A B-P_{s u c} s u c A B-s u c C D$ cluster. The transcription of the sucABCD genes, which encode subunits of 2oxoglutarate dehydrogenase $(\operatorname{sucAB})$ and succinyl-CoA synthetase (sucCD), occurs from two distinct promoter elements, $P_{s d h}$ and $P_{s u c}$. The promoter located near the start site of the sucA gene provides a relatively low constitutive level of sucABCD expression. The second promoter transcribes both the $s d h C D A B$ and the $s u c A B C D$ operon. It was demonstrated that the transcription of the sucABCD genes is primarily initiated and regulated at the upstream $s d h$ promoter, which, unlike the suc promoter, harbours a cAMP receptor protein (CRP)-binding site (Cunningham \&
Guest, 1998). All the eight genes of this cluster are upregulated in IAA-treated cells and we hypothesize that this is due to the ability of IAA to mimic cAMP action (Kline et al., 1980) even at this CRP-binding site. The induction of the $s u c D$ gene is not converted into a higher enzyme activity probably because this section of the TCA cycle is not required to be more active in IAA-treated cells. The metabolic product of SucD, succinate, can also be produced by AceA, which is activated in these cells; this might cause a feedback inhibition of SucD.

\section{Effect of IAA treatment on metabolite and cofactor levels}

To determine the effects of the IAA treatment on intermediary carbohydrate metabolism, we measured the 
Table 4. Activity of citrate synthase, isocitrate dehydrogenase and malate synthase in control and IAA-treated cells grown with different carbon sources

\begin{tabular}{|lllll|}
\hline \multirow{2}{*}{ Enzyme } & \multicolumn{3}{c|}{ Specific activity } & \\
\cline { 2 - 5 } & Carbon source & Control & IAA-treated & Ratio $^{*}$ \\
\hline Citrate synthase (GltA) & Arabinose & $0 \cdot 058 \pm 0 \cdot 007$ & $0 \cdot 091 \pm 0 \cdot 01$ & $1 \cdot 6$ \\
& Glucose & $0 \cdot 043 \pm 0 \cdot 002$ & $0 \cdot 054 \pm 0 \cdot 005$ & $1 \cdot 2$ \\
& Mannitol & $0 \cdot 051 \pm 0 \cdot 009$ & $0 \cdot 074 \pm 0 \cdot 001$ & $1 \cdot 4$ \\
& Lactose & $0 \cdot 066 \pm 0 \cdot 001$ & $0 \cdot 077 \pm 0 \cdot 006$ & $1 \cdot 2$ \\
& Glycerol & $0 \cdot 086 \pm 0 \cdot 007$ & $0 \cdot 085 \pm 0 \cdot 005$ & $0 \cdot 99$ \\
Isocitrate dehydrogenase (IcdA) & Arabinose & $0 \cdot 013 \pm 0 \cdot 003$ & $0 \cdot 023 \pm 0 \cdot 001$ & $1 \cdot 8$ \\
& Glucose & $0 \cdot 080 \pm 0 \cdot 003$ & $0 \cdot 102 \pm 0 \cdot 01$ & $1 \cdot 3$ \\
& Mannitol & $0 \cdot 051 \pm 0 \cdot 004$ & $0 \cdot 049 \pm 0 \cdot 006$ & $0 \cdot 96$ \\
& Lactose & $0 \cdot 097 \pm 0 \cdot 012$ & $0 \cdot 099 \pm 0 \cdot 009$ & $1 \cdot 0$ \\
& Glycerol & $0 \cdot 104 \pm 0 \cdot 006$ & $0 \cdot 122 \pm 0 \cdot 006$ & $1 \cdot 2$ \\
Malate synthase (AceB) & Arabinose & $0 \cdot 034 \pm 0 \cdot 005$ & $0 \cdot 048 \pm 0 \cdot 007$ & $1 \cdot 4$ \\
& Glucose & $0 \cdot 014 \pm 0 \cdot 002$ & $0 \cdot 013 \pm 0 \cdot 001$ & $0 \cdot 93$ \\
& Mannitol & $0 \cdot 019 \pm 0$ & $0 \cdot 016 \pm 0 \cdot 001$ & $0 \cdot 84$ \\
& Lactose & $0 \cdot 014 \pm 0 \cdot 002$ & $0 \cdot 016 \pm 0 \cdot 001$ & $1 \cdot 1$ \\
& Glycerol & $0 \cdot 034 \pm 0 \cdot 002$ & $0 \cdot 041 \pm 0 \cdot 003$ & $1 \cdot 2$ \\
\hline
\end{tabular}

${ }^{*}$ The unit of enzyme activity is $\mu \mathrm{mol} \mathrm{min}^{-1}$ (mg protein $)^{-1}$.

$\dagger$ Ratio calculation was based on the control condition. The values reported in the table are the means $\pm \mathrm{SD}$ of at least five measurements.

intracellular levels of acetyl-CoA and the extracellular level of acetate and pyruvate. The concentrations of acetyl-CoA $(1 \cdot 0 \pm 0 \cdot 1 \mathrm{mM}$ for control cells, $2 \cdot 7 \pm 0 \cdot 3 \mathrm{mM}$ for IAAtreated cells) and acetate $(0 \cdot 480 \pm 0 \cdot 021 \mathrm{mM}$ for control cells, $0.692 \pm 0.060 \mathrm{mM}$ for IAA-treated cells) increased while the level of pyruvate excreted $(0.066 \pm 0.003 \mathrm{mM}$ for control cells, $0 \cdot 046 \pm 0 \cdot 002 \mathrm{mM}$ for IAA-treated cells) decreased. In addition, the determination of the steadystate internal redox state, as reflected by the $\mathrm{NADH} / \mathrm{NAD}^{+}$ ratio, showed that IAA treatment led to a $1 \cdot 3$-fold decrease of this ratio after $30 \mathrm{~min}$ (Fig. 2). This effect was much higher after $2 \mathrm{~h}$, when the $\mathrm{NADH} / \mathrm{NAD}^{+}$ratio was even lower $(3 \cdot 2$-fold decrease). The total dinucleotide pool also increased up to $40 \%$ in IAA-treated cells (data not shown).

\section{DISCUSSION}

The aim of this study was to investigate the response of E. coli cells to IAA treatment. To evaluate the global effects triggered by IAA addition, we measured the mRNA abundance, assayed 34 enzyme activities and determined the level of five diagnostic metabolites. In many cases the response measured at the mRNA level was in agreement with the corresponding enzyme activity, as shown by the genes and enzymes involved in the TCA cycle and glyoxylate shunt, both up-regulated in IAA-treated cells. This response results in the coordinate changes observed for cellular metabolite production such as acetyl-CoA, $\mathrm{NAD}^{+}$, $\mathrm{NADH}$, acetate and pyruvate.
The determination of metabolite levels revealed that IAAtreated cells synthesized higher acetyl-CoA levels as compared to the control cells. In response to this perturbation, treated cells conveyed acetyl-CoA to the phosphotransacetylase (Pta)-acetate kinase (Ack) pathway (Table 3, Fig. 1), producing and excreting more acetate in the medium. The increased acetate production was accomplished by excreting less pyruvate into the medium, in agreement with the well-known increased pyruvate production in mutants with a defective Pta-AckA pathway (Chang et al., 1999).

The higher acetate excretion observed in treated cells is an effect caused by the imbalance between the uptake of the substrate and the demand for biosynthesis and energy production. This adjustment functions as a safety valve, as proposed by Holms (1996) from stoichiometric flux analysis of the central metabolic pathways in E. coli. The accumulation of acetate observed in the extracellular milieu of IAA-treated cells might be of evolutionary advantage: it could increase the metabolic flexibility of these cells during adaptation to a changing environment. In particular, during the transition to stationary phase, the cells might well undergo a metabolic switch: instead of excreting acetate, they could reabsorb it, activate it to acetyl-CoA by means of Acs, and utilize the acetate to generate energy and biosynthetic components via the TCA cycle and the glyoxylate shunt, respectively. Most of the metabolic genes differentially expressed in IAA-treated cells are catabolite-sensitive genes: their transcription is subject to cAMP-dependent catabolite repression. Among them we found the TCA cycle genes gltA, sdhB, sucA and $s u c D$, in 


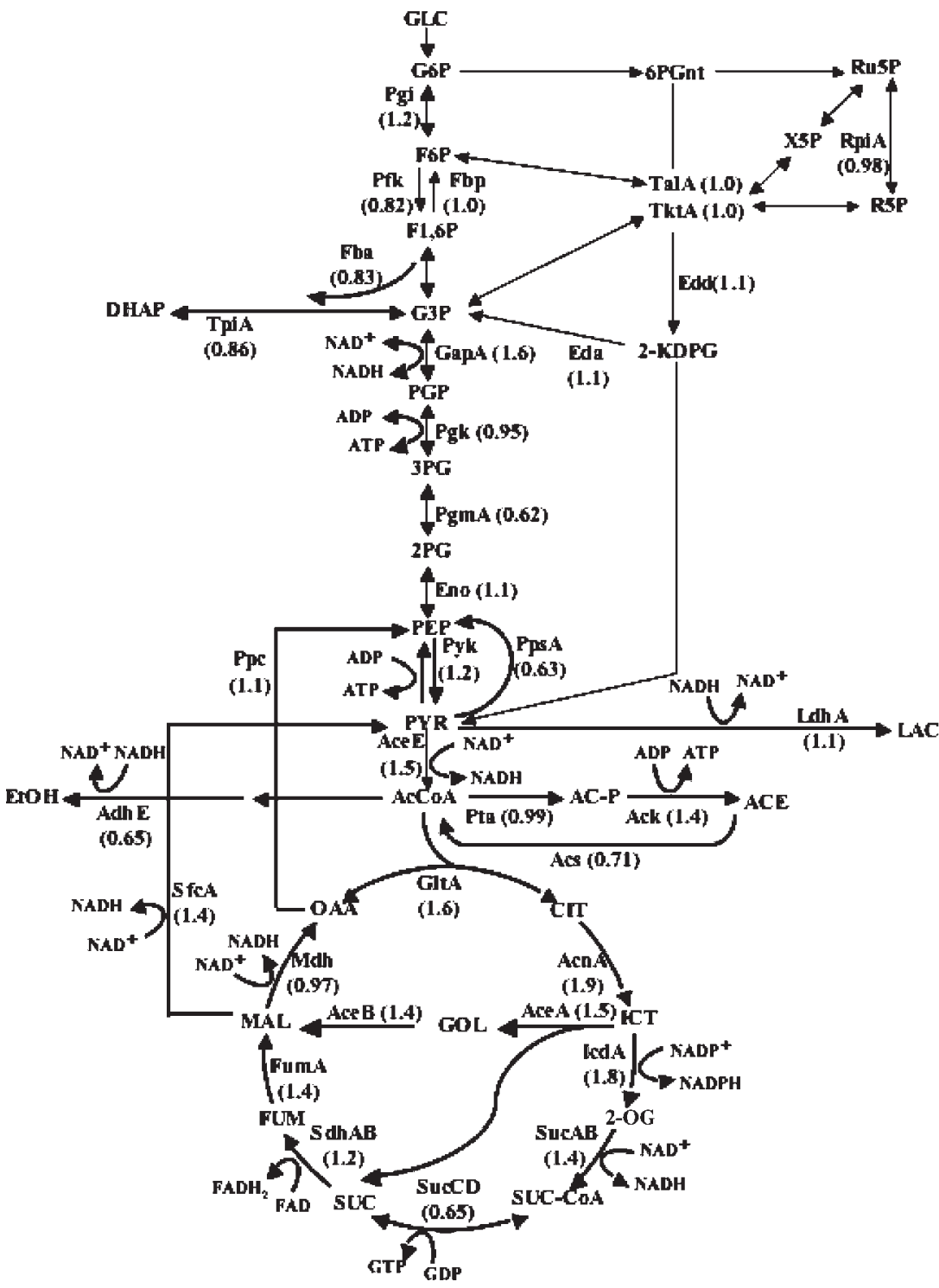

Fig. 1. Activities of central metabolic pathway enzymes in IAA-treated cells compared with those of control cells. The numbers beside enzyme names represent ratios based on the control condition and are the mean of five repeated experiments. Abbreviations: GLC, glucose; G6P, glucose 6-phosphate; F6P, fructose 6-phosphate; F1,6P, fructose 1,6-phosphate; G3P, glyceraldehyde 3phosphate; PGP, 3-phospho-D-glyceroyl phosphate; 3PG, 3-phosphoglycerate; 2PG, 2-phosphoglycerate; PEP, phosphoenolpyruvate; PYR, pyruvate; LAC, lactate; AcCoA, acetyl-CoA; AC-P, acetyl phosphate; ACE, acetate; $\mathrm{EtOH}$, ethanol; CIT, citrate; ICT, isocitrate; 2-OG, 2-oxoglutarate; SUC-CoA, succinyl-CoA; SUC, succinate; FUM, fumarate; $\mathrm{MAL}$, malate; OAA, oxalacetate; GOL, glyoxylate; 6PGnt, 6-phospho-D-gluconate; Ru5P, D-ribulose-5-phosphate; R5P, D-ribose-5phosphate; X5P, D-xylulose-5-phosphate; 2KDPG, 2-keto-3-deoxy-6-phosphogluconate; DHAP, dihydroxyacetone phosphate.

the gltA-sdhCDAB-sucAB-sucCD gene cluster, and the $c y o D$ gene, encoding one component of the low-affinity terminal oxidase cytochrome $b o_{3}$, the respiratory system that is derepressed in aerobic conditions.

It is known that the cytochrome $b o_{3}$ respiratory system, as well as cytochrome $b d$, is necessary to regenerate $\mathrm{NAD}^{+}$ from $\mathrm{NADH}$ and to eliminate excess reducing equivalents (Garcia-Horsman et al., 1994). Accordingly, we found that the $\mathrm{NADH} / \mathrm{NAD}^{+}$ratio after IAA treatment dropped to values roughly threefold lower than those found for control cells. This effect plays an important role in IAA-treated cells avoiding repression of NADH-producing enzymes, specially two of the TCA cycle's rate-controlling enzymes (IcdA and SucAB), subject to a product inhibition mechanism (de Graef et al., 1999). The lower NADH/NAD ${ }^{+}$ratio correlates with the reduced production of the less energyefficient fermentative gene $a d h E$ and the repression of the alcohol dehydrogenase activity. Indeed, Leonardo et al. (1996), by directly measuring the NADH-NAD ${ }^{+}$levels and by monitoring the expression of adhE, demonstrated that this $E$. coli gene is induced under high-NADH/NAD ${ }^{+}$ conditions and repressed under low-NADH/NAD ${ }^{+}$conditions. The decrease of $\mathrm{NADH} / \mathrm{NAD}^{+}$also prevents the inactivation of another enzyme subject to catabolite repression, the AceE enzyme in the pyruvate dehydrogenase complex (required for the oxidative carboxylation of pyruvate to produce $\mathrm{CO}_{2}$ and acetyl-CoA). The induction of the pyruvate dehydrogenase complex observed in treated cells correlated with the reduced conversion of pyruvate through phosphoenolpyruvate synthase (Pps) (Table 3).

In addition, IAA-treated cells induced the synthesis of genes encoding protective molecules such as molecular chaperones (yrfI, dnaK, yfhE and $h t p G$ gene products) to prevent 


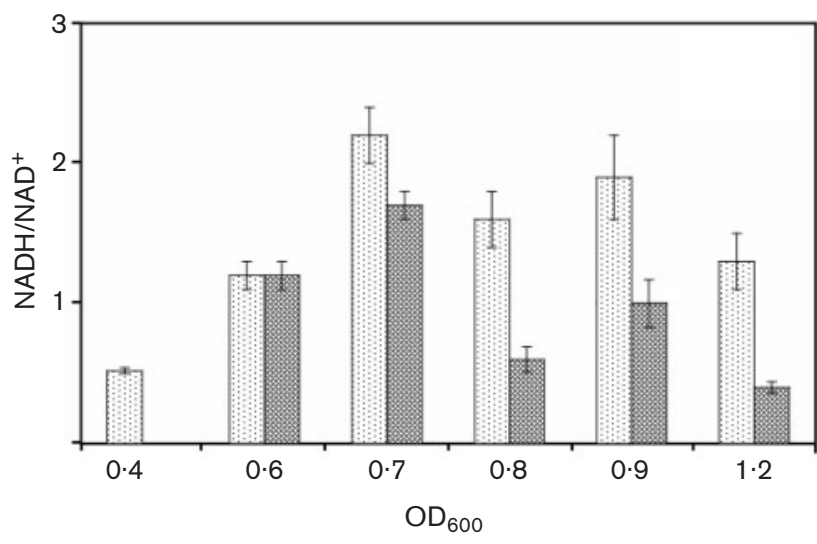

Fig. 2. $\mathrm{NADH} / \mathrm{NAD}^{+}$ratio of control (light columns) and treated (dark columns) cells versus $\mathrm{OD}_{600}$. Exponentially growing cultures $\left(\mathrm{OD}_{600} 0.6\right)$ were treated with $0.5 \mathrm{mM}$ IAA for $2 \mathrm{~h}\left(\mathrm{OD}_{600} 1 \cdot 2\right)$ and samples taken at intervals for analysis. The values are the means $\pm S D$ of at least five independent measurements.

the damage caused to many biological molecules by reactive oxygen species, such as hydrogen peroxide, produced during aerobic respiration.

Finally, the up-regulation of the two central backbones of metabolism, the TCA cycle and the glyoxylate shunt, provides cofactor regeneration and building blocks for amino acid biosynthesis (Akashi \& Gojobori, 2002). We found that $10 \%$ of the genes showing altered expression levels in IAA-treated cells were involved in amino acid biosynthesis: they were all up-regulated. Among them we found genes encoding enzymes required for the biosynthesis of valine and leucine, derived from intermediates produced in late steps of glycolysis, and for isoleucine and proline, derived from oxalacetate and 2-oxoglutarate, two precursor metabolites produced in the TCA cycle. We thus hypothesize that the increased expression of the amino acid biosynthetic genes is indicative of a nitrogen-sufficient condition.

Taken globally, these results suggest that IAA mimics cAMP action in the catabolite derepression of many metabolic genes (i.e. $\operatorname{art} Q, r b s C, m l c, g l p K$, TCA cycle genes and $c y o D)$. It has been described that the ilvB gene (Williams, 1986) and the araBAD operon (Ebright \& Beckwith, 1985) were specifically induced by IAA. Those reports and our data confirm that IAA cannot replace cAMP at all known sites of action, as we did not find that all catabolite-repressed genes are induced. Further studies to investigate a physical interaction between IAA and the ara operon transcriptional regulators might explain this aspect. We cannot say if the catabolite gene activator protein CAP (the crp gene product) is directly involved in the modulation of the catabolite repression observed in IAA-treated cells. Regulation, exerted by the CAMP-CRP complex in catabolite repression, is well established. However, several lines of evidence indicate that the cAMP-CRP complex may not be the only regulator involved in catabolite repression (Crasnier, 1996). In addition, the induction of the arabinose operon in cya mutant strains does not require CRP, as shown by Ebright \& Beckwith (1985). Thus IAA might act through a different mechanism, for example via interaction with a new mediator.

IAA functions as a regulator of cellular metabolism in E. coli: it co-ordinates changes in carbon and nitrogen metabolism that are used by the cells to maintain metabolic homeostasis and to optimize energy production during aerobic life.

De Melo et al. (2004) found that human tumour cells treated with IAA showed activated flux of substrates through the TCA cycle and a decreased $\mathrm{NADH} / \mathrm{NAD}^{+}$ratio, showing a striking similarity with our data, in a quite distant system. The alterations triggered by IAA in the main metabolic pathways of bacterial, yeast and human cells leave an open question. Can some of the effects observed in higher organisms be explained by the action of IAA on cytoplasmic organelles? Does IAA act in E. coli as a hormone, as a second messenger or via physical interaction with DNA (Ebright \& Wong, 1981)?

Our results provide the first extensive analysis of the action of IAA on the bacterial metabolism, allowing similar kinds of questions to be more precisely addressed.

\section{ACKNOWLEDGEMENTS}

This work was supported by, the European Union, INCO-DEV SONGLINES grant, project ICA4-CT-2001-10059, and by Italian MIUR, project FIRB RBNE018BHE. We thank C. Sole and R. Vito for technical assistance.

\section{REFERENCES}

Akashi, H. \& Gojobori, T. (2002). Metabolic efficiency and amino acid composition in the proteomes of Escherichia coli and Bacillus subtilis. Proc Natl Acad Sci U S A 99, 3695-3700.

Alleanza, P. \& Lessie, G. T. (1982). Pseudomonas cepacia mutants blocked in Entner-Doudoroff pathway. J Bacteriol 150, 1340-1347.

Artigas, F., Sunol, C., Tusell, J. M., Martinez, E. \& Gelpi, E. (1985). Comparative ontogenesis of brain tryptamine, serotonin, and tryptophan. J Neurochem 44, 31-37.

Bridger, W. A., Ramaley, R. F. \& Boyer, P. D. (1969). Succinyl coenzyme A synthase from Escherichia coli. Methods Enzymol 13, 70-71.

Brown, T. D., Jones-Mortimer, M. C. \& Kornberg, H. L. (1977). The enzymatic interconversion of acetate and acetyl-coenzyme A in Escherichia coli. J Gen Microbiol 102, 327-336.

Chang, D. E., Shin, S., Rhee, J. S. \& Pan, J. G. (1999). Acetate metabolism in a pta mutant of Escherichia coli W3110: importance of maintaining acetyl coenzyme A flux for growth and survival. J Bacteriol 181, 6656-6663. 
Chohnan, S., Izawa, H., Nishihara, H. \& Takamura, Y. (1998). Changes in size of intracellular pools of coenzyme $\mathrm{A}$ and its thioesters in Escherichia coli K-12 cells to various carbon sources and stresses. Biosci Biotechnol Biochem 62, 1122-1128.

Clark, D. \& Cronan, J. E. (1980). Escherichia coli mutants with altered control of alcohol dehydrogenase and nitrate reductase. J Bacteriol 141, 177-183.

Cooper, R. A. \& Kornberg, H. L. (1966). Phosphoenolpyruvate synthase. Methods Enzymol 8, 309-314.

Crasnier, M. (1996). Cyclic AMP and catabolite repression. Res Microbiol 147, 479-482.

Cunningham, L. \& Guest, J. R. (1998). Transcription and transcript processing in the $s d h C D A B-s u c A B C D$ operon of Escherichia coli. Microbiology 144, 2113-2123.

D'alessio, G. \& Josse, J. (1966). Phosphoglycerate kinase and phosphoglyceromutase from Escherichia coli. Methods Enzymol 42, 139-144.

Davies, P. J. (1995). Plant Hormones. Dordrecht, The Netherlands: Kluwer.

De Felice, M., Griffo, G., Lago, T. C., Limanuro, D. \& Ricca, E. (1988). Detection of the acetolactate synthase isoenzyme I and III of Escherichia coli K-12. Methods Enzymol 166, 241-244.

de Graef, R. M., Alexeeva, S., Snoep, J. L. \& Teixeira de Mattos, J. (1999). The steady-state internal redox state (NADH/NAD) reflects the external redox state and is correlated with catabolic adaptation in Escherichia coli. J Bacteriol 181, 2351-2357.

De Melo, M. P., Pithon-Curi, T. C. \& Curi, R. (2004). Indole-3acetic acid increases glutamine utilization by high peroxidase activity-presenting leucocytes. Life Sci 75, 1713-1725.

Dixon, G. H. \& Kornberg, H. L. (1969). Malate synthase from baker's yeast. Methods Enzymol 13, 633-634.

Donahue, J. L., Bownas, J. L., Niehaus, W. G. \& Larson, T. J. (2000). Purification and characterization of $g l p X$-encoded fructose 1,6bisphosphatase, a new enzyme of the glycerol 3-phosphate regulon of Escherichia coli. J Bacteriol 182, 5624-5627.

Ebright, R. H. \& Beckwith, J. (1985). The catabolite gene activator protein (CAP) is not required for indole-3-acetic acid to activate transcription of the araBAD operon of Escherichia coli K-12. Mol Gen Genet 201, 51-55.

Ebright, R. H. \& Wong, J. R. (1981). Mechanism of transcriptional action of cyclic AMP in Escherichia coli: entry into DNA to disrupt DNA secondary structure. Proc Natl Acad Sci U S A 78, 4011-4015.

Eikmanns, B. J. (1992). Identification, sequence analysis and expression of Corynebacterium glutamicum gene cluster encoding the three glycolytic enzymes glyceraldehyde-3-phosphate dehydrogenase, 3phosphoglycerate kinase, and triosephosphate isomerase. J Bacteriol 174, 6076-6086.

Fansler, B. \& Lowenstein, J. M. (1969). Aconitase from pig heart. Methods Enzymol 13, 26-28.

Garcia-Horsman, J. A., Barquera, B., Rumbley, J., Ma, J. \& Gennis, R. B. (1994). The superfamily of heme-copper respiratory oxidases. J Bacteriol 176, 5587-5600.

Hill, R. L. \& Bradshaw, A. R. (1969). Fumarase. Methods Enzymol 13, 91-93.

Holms, H. (1996). Flux analysis and control of the central metabolic pathways in Escherichia coli. FEMS Microbiol Rev 19, 85-116.

Hove-Jensen, B. \& Maigaard, M. (1993). Escherichia coli rpiA gene encoding ribose phosphate isomerase A. J Bacteriol 175, 5628-5635.

Insland, M. D., Wei, B. Y. \& Kadner, R. J. (1992). Structure and function of the $u h p$ genes for the sugar phosphate transport system in Escherichia coli and Salmonella typhimurium. J Bacteriol 174, 2754-2762.

Josephson, L. B. \& Fraenkel, D. G. (1969). Transketolase mutants of Escherichia coli. J Bacteriol 100, 1289-1295.

King, T. E. (1969). Preparation of succinate dehydrogenase and reconstitution of succinate oxidase. Methods Enzymol 10, 322-331.

Kitto, B. G. (1969). Intra- and extramitochondrial malate dehydrogenase from chicken and tuna heart. Methods Enzymol 13, 106-107.

Kline, L. E., Brown, C. S., Bankaitis, V., Montefiori, D. C. \& Craig, K. (1980). Metabolite gene regulation of the L-arabinose operon in Escherichia coli with indoleacetic acid and other indole derivatives. Proc Natl Acad Sci U S A 77, 1768-1772.

Kochhar, S., Chuard, N. \& Hottinger, H. (1992). Glutamate 264 modulates the $\mathrm{pH}$ dependence of the $\mathrm{NAD}^{+}$-dependent D-lactate dehydrogenase. J Biol Chem 267, 20298-20301.

Lemcke, K., Prinsen, E., Van Onckelen, H. \& Schmülling, T. (2000). The ORF8 gene product of Agrobacterium rhizogenes TL-DNA has tryptophan 2-monooxygenase activity. Mol Plant Microbe Interact 13, 787-790.

Leonardo, M. R., Dailly, Y. \& Clark, D. P. (1996). Role of NAD in regulating the adhE gene of Escherichia coli. J Bacteriol 178, 6013-6018.

Ling, K. H., Paetkau, V., Marcus, F. \& Lardy, H. A. (1969). Phosphofructokinase. Methods Enzymol 9, 425-429.

Livak, K. J. \& Schmittgen, T. D. (2001). Analysis of relative gene expression data using real-time quantitative PCR and the $2^{\Delta \Delta C T}$ method. Methods 25, 402-408.

McFadden, A. B. (1969). Isocitrate lyase. Methods Enzymol 13, 163-165.

Muro-Pastor, M. I. \& Florencio, F. J. (1992). Purification and properties of NADP-isocitrate dehydrogenase from the unicellular cyanobacterium Synechocystis sp. PCC 6803. Eur J Biochem 203, 99-105.

Pagnussat, G. C., Lanteri, M. L. \& Lamattina, L. (2003). Nitric oxide and cyclic GMP are messengers in the indole acetic acid-induced adventitious rooting process. Plant Physiol 132, 1241-1248.

Patnaik, R., Roof, W. D., Yong, F. R. \& Liao, J. C. (1992). Stimulation of glucose catabolism in Escherichia coli by potential futile cycle. J Bacteriol 174, 7527-7532.

Pruss, B. M. \& Wolfe, A. J. (1994). Regulation of acetyl phosphate synthesis and degradation, and control of flagellar expression in Escherichia coli. Mol Microbiol 12, 973-984.

Prusty, R., Grisafi, P. \& Fink, G. R. (2004). The plant hormone indoleacetic acid induces invasive growth in Saccharomyces cerevisiae. Proc Natl Acad Sci U S A 101, 4153-4157.

Reed, L. J. \& Mukherjee, B. B. (1969). $\alpha$-Ketoglutarate dehydrogenase complex from Escherichia coli. Methods Enzymol 13, 55-61.

Reithel, F. J. (1966). Phosphoglucose isomerase. Methods Enzymol 9, 565-569.

Rutter, J. W., Hunsley, J. R., Groves, W. E., Calder, J., Rajkumar, T. V. \& Woodfin, B. M. (1969). Fructose diphosphate aldolase. Methods Enzymol 9, 479-498.

Schartz, E. R., Old, L. O. \& Reed, L. J. (1968). Regulatory properties of pyruvate dehydrogenase from Escherichia coli. Biochem Biophys Res Commun 31, 495-500.

Shin, D., Lim, S., Seok, Y. J. \& Ryu, S. (2001). Heat shock RNA polymerase $\left(\mathrm{E} \sigma^{32}\right)$ is involved in the transcription of $m l c$ and crucial for induction of the Mlc regulon by glucose in Escherichia coli. J Biol Chem 276, 25871-25875.

Skarstedt, M. T. \& Silverstein, E. (1976). Escherichia coli acetate kinase mechanism studied by net initial rate, equilibrium, and independent isotopic exchange kinetics. J Biol Chem 251, 6775-6783. 
Stols, L. \& Donnelly, M. I. (1997). Production of succinic acid through overexpression of $\mathrm{NAD}^{+}$-dependent malic enzyme in an Escherichia coli mutant. Appl Environ Microbiol 63, 2695-2701.

Takahashi, K., Kasai, K. \& Ochi, K. (2004). Identification of the bacterial alarmone guanosine $5^{\prime}$-diphosphate $3^{\prime}$-diphosphate (ppGpp) in plants. Proc Natl Acad Sci U S A 101, 4320-4324.

Tchola, O. \& Horecker, B. L. (1966). Transaldolase. Methods Enzymol 9, 499-505.

Valentine, W. N. \& Tanaka, K. R. (1966). Pyruvate kinase: clinical aspects. Methods Enzymol 9, 468-473.

Weissenborn, D. L., Wittekindt, N. \& Larson, T. J. (1992). Structure and regulation of the $g l p F K$ operon encoding glycerol diffusion facilitator and glycerol kinase of Escherichia coli K-12. J Biol Chem 267, 6122-6131.

Weitzman, P. D. J. (1969). Citrate synthase from Escherichia coli. Methods Enzymol 13, 22-26.
Westhead, E. W. (1966). Enolase from yeast and rabbit muscle. Methods Enzymol 9, 671-679.

Williams, A. L. (1986). Regulation of acetohydroxy acid synthase activities in Escherichia coli $\mathrm{K}-12$ by small metabolites. Biochim Biophys Acta 866, 15-18.

Wohl, R. C. \& Markus, G. (1972). Phosphoenolpyruvate carboxylase of Escherichia coli. Purification and some properties. J Biol Chem 247, 5785-5792.

Zhang, Z., Yu, J. \& Stanton, R. C. (2000). A method for the determination of pyridine nucleotides using a single extract. Anal Biochem 285, 163-167.

Zimmer, W., Wesche, M. \& Timmermans, L. (1998). Identification and isolation of the indole-3-pyruvate decarboxylase gene from Azospirillum brasilense Sp7: sequencing and functional analysis of the gene locus. Curr Microbiol 36, 327-331. 